\title{
Lessons Learnt from the Second Generation of Anti-Amyloid Monoclonal Antibodies Clinical Trials
}

\author{
Angela Tian Hui Kwan ${ }^{a, b, c}$ Saman Arfaie ${ }^{b, c, d, e}$ Joseph Therriault ${ }^{b, c, f}$ \\ Pedro Rosa-Netob, c, f Serge Gauthier ${ }^{b, c, f}$ \\ aDepartment of Chemical \& Physical Sciences, University of Toronto, Toronto, ON, Canada; ${ }^{\mathrm{b} T r a n s l a t i o n a l}$ \\ Neuroimaging Laboratory, The McGill University Research Centre for Studies in Aging, Douglas Mental Health \\ University Institute, McGill University, Montreal, QC, Canada; 'Department of Neurology and Neurosurgery, McGill \\ University, Montreal, QC, Canada; ${ }^{d}$ Faculty of Medicine, McGill University, Montreal, QC, Canada; ${ }^{\text {Department }}$ of \\ Molecular Cell Biology, University of California, Berkeley, CA, USA; 'Department of Psychiatry, McGill University, \\ Montreal, QC, Canada
}

\section{Keywords}

Aducanumab $\cdot$ Alzheimer disease $\cdot$ Amyloid-beta $\cdot$ APOE4 . Clinical trials · Crenezumab · DIAN-TU · Disease-modifying therapy · Gantenerumab · Solanezumab

\section{Abstract \\ Background: Alzheimer disease (AD) is a chronic neurode- generative disorder with complex pathophysiology that af- fects over 50 million people worldwide. Most drug therapies, to date, have focused on targeting the amyloid-beta $(A \beta)$ pathway, but clinical outcomes of anti-A $\beta$ antibodies have been unsuccessful and unable to meet their primary end- points. Similar trends have also been observed in treatments that target the tau pathway. Summary: This paper reviews recent anti-A $\beta$ passive monotherapies, since Bapineuzum- $a b$, that have progressed to phase 3 clinical trials. Specifi- cally, we discuss the 4 clinical trial programs of Solanezumab (targets $A \beta$ monomers), Aducanumab (targets $A \beta$ oligomers and plaques), Crenezumab (targets $A \beta$ oligomers), and Gan- tenerumab (targets $A \beta$ fibrils) which are all exogenous monoclonal antibodies. We conclude with potential reasons}

for why they have not met their primary endpoints and discuss lessons learnt from these trials. Key Message: Future disease-modifying trials (DMTs) for AD should be conducted in asymptomatic, $A \beta$-positive individuals. Moreover, potential additive and/or synergistic benefits focusing on anti-A $\beta$ and anti-tau drug combinations merit further investigation.

(c) 2020 S. Karger AG, Basel

\section{Introduction: Alzheimer Disease}

According to the biological framework, Alzheimer disease $(\mathrm{AD})$ is defined as a neurodegenerative disorder characterized by the accumulation of amyloid-beta $(\mathrm{A} \beta)$ plaques and tau neurofibrillary tangles, which are associated with neurodegeneration and cognitive decline [1]. $\mathrm{AD}$ remains the most common cause of dementia globally, and the prevalence estimates of biological AD exceed that of amnestic dementia $[2,3]$. Following these preclinical and prodromal phases, the subsequent clinical phase spans from 8 to 10 years [4]. Recent findings have suggested that $\mathrm{AD}$ has an estimated prevalence of $10-30 \%$ in 
populations aged 65 or older, with an incidence rate of $1-3 \%$ [4]. Increasing age is the biggest risk factor associated with developing AD and other dementias [5]. This is problematic because the center for disease control has estimated an increase in the people over the age of 65 from 420 million, in 2000, to 1 billion by 2030 [6]. In addition to its wide prevalence, there is a significant impact on the direct cost of AD to society. A total of approximately $\$ 290$ billion were spent in 2019 alone on hospice services, health care, and long-term care for patients aged 65 and over with dementia [3].

To date, only a handful of $\mathrm{AD}$ treatments have been FDA approved, which include the acetylcholinesterase inhibitors donepezil, galantamine, and rivastigmine as well as the noncompetitive N-Methyl-D-Aspartate receptor antagonist memantine $[7,8]$. Their utilities are limited as they only provide partial symptomatic relief to patients with mild-to-severe dementia and are unable to alter the course of $\mathrm{AD}$ progression [8]. As a result, efforts have been concentrated on an expansion towards developing disease-modifying therapies (DMTs) that can change the underlying pathophysiology of $\mathrm{AD}$ [9]. While there have been studies on over $200 \mathrm{AD}$ drug candidates, most of these therapeutic agents have either failed or were abandoned during clinical trials $[10,11]$.

Passive $A \beta$ immunotherapies have been successful in animal models of $\mathrm{AD}$ and showed promise in reversing cognitive impairment and reducing brain pathology through microglial stimulation or prevention of $A \beta$ aggregation $[12,13]$. These successes prompted researchers to progress to human clinical trials. This paper reviews recent passive $A \beta$ immunotherapies, which consists of the phase 3 trials of DMTs Solanezumab, Aducanumab, Crenezumab, and Gantenerumab. We review their mechanisms of action, trial results, and potential reasons for failure to meet primary endpoints and discuss future directions for anti-amyloid therapeutic mechanisms in $\mathrm{AD}$.

\section{Disease-Modifying Therapies}

Solanezumab (LY2062430): Amyloid Remover and Aggregation Inhibitor (Anti-A $\beta$, Targets Monomers, and Disease-Modifying Therapy)

Developed by Eli Lilly \& Co., Solanezumab is a humanized monoclonal immunoglobulin G1 (IgG1) antibody that targets neurotoxic $A \beta$ peptides in mild-tomoderate AD brains [14]. Specifically, the drug binds with picomolar affinity to an epitope within the mid-domain of $A \beta$ in its soluble monomeric state [15]. However, it excludes preformed deposited $A \beta$ or $A \beta$ plaques [15]. The epitope - characterized by A $\beta$ residues 16-26 (KLVFFAEDVGS) - is also the nucleation site for $A \beta$ oligomerization of the two main isoforms: $A \beta 40$ and $A \beta 42$ [16]. Consequently, by binding to this region, the extensive contacts formed between Solanezumab and the A $\beta$ peptide inhibit the process of $A \beta$ aggregation, leading to the sequestration of other free monomers [17].

Passive immunization of this antibody alters central nervous system (CNS) and plasma $A \beta$ clearance $[16,18]$. It causes a reduction in $A \beta$ burden, which is hypothesized to occur through the peripheral sink mechanism of action $[16,18]$. Majority of the drug remains in the periphery post-intravenous administration. It binds to soluble $A \beta$ peptides within the bloodstream and sequesters them into an immune complex [18]. As a result, the plasma $A \beta$ concentration decreases as the formed immune complexes are subsequently cleared from the circulation [18]. The equilibria between the $A \beta$ oligomers, aggregates, and plaques in the CNS then shift to produce soluble monomers, which become transported across the blood-brain barrier to restore the reduced plasma A $\beta$ level [18]. The net efflux of brain $A \beta$ monomers - mediated by the lowdensity lipoprotein receptor 1 - in turn elevates $A \beta$ clearance in the CNS [18]. Accordingly, the CNS A $\beta$ level decreases, ultimately lowering $A \beta$-related cellular toxicity and $A \beta$ pathology [18].

Given its phase 2 results, where the drug's mode of action was established and circumstantial evidence on its effect on $A \beta$ plaques was found, phase 3 clinical trials were initiated. Precisely, this included tworandomized, double-blinded expeditions with 1,012 (506 per study group) and 1,040 (521 in the Solanezumab group and 519 in the placebo group) mild-to-moderate $\mathrm{AD}$ patients, who received either placebo or Solanezumab intravenously at $400 \mathrm{mg} / \mathrm{month}$ for 80 weeks [15]. The co-primary outcomes assessed cognitive impairment and competence in activities of daily living, measured by the 11item cognitive subscale of the Alzheimer's Disease Assessment Scale (ADAS-Cog1 1; range 0-70 with higher scores denoting greater cognitive impairment) and the Alzheimer's Disease Cooperative Study-Activities of Daily Living (ADCS-ADL; range 0-78 with lower scores denoting worse functioning) scale, respectively $[15,19]$. Moreover, the secondary cognitive outcomes involved the Clinical Dementia Rating - Sum of Boxes (CDR-SB), Mini-Mental State Examination (MMSE), Neuropsychiatric Inventory (NPI), EuroQol 5Dimensional Health-related Quality of Life Scale Proxy version (EQ-5D Proxy) scale, Resource Utilization in Dementia Lite (RUD-Lite) 
scale, and Quality of Life in Alzheimer's Disease (QOLAD) scale score changes [19]. Secondary biomarker outcomes included evaluation of plasma and cerebrospinal fluid (CSF) A $\beta$ levels, assessment of CSF tau and phospho-tau levels, MRI brain volumetric measurements, and 18F-florbetapir-Positron Emission Tomography (PET) to determine the presence of $A \beta$ accumulation [19].

In expedition 1 , the results showed that patient ADASCog 11 and ADCS-ADL scores remained unchanged after treatment with Solanezumab, signifying failure to improve cognition or functional ability [19]. The mean difference between the Solanezumab and placebo groups in the change from baseline to week 80 was -0.8 and -0.4 points, respectively [19]. Furthermore, no significant treatment-associated differences were observed in the score changes of CDR-SB $(p=0.51)$, MMSE $(p=0.06)$, and NPI $(p=0.29)$ [19]. No significant differences between treatment and placebo groups were also observed for the EQ-5D, RUD-Lite, and QOL-AD scales [15]. However, when the mild AD patients - who have a MMSE score of 20-26 - were analyzed separately, improvements in cognitive function were detected via the ADAS-Cog11 and 14-item cognitive subscale of the Alzheimer's Disease Assessment Scale (ADAS-Cog14; range 0-90 with higher scores denoting greater impairment) [19]. When assessing secondary biomarker outcomes, plasma $A \beta$ assessments revealed that levels of $A \beta 40$ and $A \beta 42$ both increased due to the drug's peripheral sink activity in the Solanezumab group $(p<0.001)$ [19]. Consequently, the CNS A $\beta$ level becomes reduced. Likewise, CSF A $\beta$ assessments revealed a reduction in the concentration of free $A \beta 40$, an increase in total $A \beta 40$, and an increase in total A $\beta 42$ had occurred (between-group difference: $p=0.01$, $p=0.002$, and $p<0.001$ ) [19]. Both results demonstrated successful target engagement of Solanezumab with soluble CNS A $\beta$ peptides. The CSF data revealed $A \beta$ shifting within the central compartment, which may have led to $A \beta$ efflux into the periphery or disturbance of the fibrillar-soluble $A \beta$ equilibrium that ultimately reduced soluble brain $A \beta$. Though, the hippocampal and total brain volumes decreased, just like for placebo, since the antibody was not designed to target $A \beta$ fibrils [19]. No significant differences were seen with $A \beta$ accumulation either, as measured via $18 \mathrm{~F}-$ florbetapir-PET. Last, for either cohort, both CSF tau and phospho-tau levels remained unaltered.

For expedition 2, the results also showed that Solanezumab failed to improve patient ADAS-Cog and ADCSADL scores. The mean difference between the Solanezumab and placebo groups in the change from baseline to week 80 was -1.3 and -1.6 points, respectively [19]. As well, ADAS-Cog14 scores were measured for mild and moderate AD patients as -1.7 and -1.5 , respectively ( $p=$ $0.06, p=0.26$ ) [19]. Thus, neither study reached their primary outcomes. In terms of the secondary outcomes, a favorable difference of 0.8 points was observed for MMSE $(p=0.01)$ [19]. Also, mild AD patients experienced a significant treatment effect on ADCS-ADL at 2.3 points $(p=0.04)$ and moderate AD patients with MMSE at 1.0 $(p=0.04)$ [19]. In terms of safety, the incidence of Amyloid-Related Imaging Abnormalities (ARIAs) was measured to be $0.9 \%$ for Solanezumab and $0.4 \%$ for placebo for Alzheimer's Related Imaging Abnormality-Edema (ARIA-E) $(p=0.27)$ and 4.9 and $5.6 \%$ for Alzheimer's Related Imaging Abnormality Microhemorrhages (ARIA-H) $(p=0.49)$, respectively [19].

Given the positive findings for the mild AD cohort in both expeditions, the data were combined for a secondary pooled analysis. A significant effect on cognitive performance was observed due to the Solanezumab therapy, as measured by ADAS-Cog 14, with expedition 1 being the primary driver of this improvement. However, the ADCSADL scores remained unchanged; though, a positive trend was observed [15]. As well, through the use of the $\left[{ }^{18} \mathrm{~F}\right]$ Florbetapir PET amyloid imaging agent, no significant reduction of $A \beta$ deposit levels was seen. Moreover, an increase in total (bound and unbound) CSF A $\beta 40$ and $A \beta 42$, a decrease in free CSF $A \beta 40$, and an increase in bound plasma $A \beta$ were detected. Finally, no changes in CSF p-tau levels and no changes in hippocampal or whole brain volume were observed [15]. These findings aligned with results from phase 2 except a significant increase in free CSF $A \beta 42$ did not occur in these expeditions.

Based on the data from this secondary pooled analysis, expedition 3 was initiated. This phase 3 trial focused on examining the effects of Solanezumab in 2,129 mild AD dementia patients (MMSE score of 20-26) who suffered from brain $A \beta$ burden, as identified by means of Florbetapir PET or CSF A $\beta 42$ measurements [20]. The same dosing regimen and duration used in the previous 2 expeditions were implemented. The co-primary endpoints includedADAS-Cog14andADCS-iADL.ForADAS-Cog14, the results showed that the mean change from baseline was 6.65 and 7.44 for the Solanezumab and placebo groups, respectively [20]. No significant between-group difference was observed at week 80 ( -0.80 difference, $p=$ $0.10)$. For ADCS-iADL, there was a decrease in the ADCSiADL score for both trial groups, indicating worsening of patient competence [20]. With regards to the secondary outcomes, a decrease was seen for MMSE (-3.17, Solan- 
ezumab group and -3.66 , placebo), ADCS-ADL, and iADRS, along with an increase (worsening) in the scores on the FAQ and CDR-SB [20]. Moreover, the safety measurements revealed adverse cerebral edema or effusion lesions in 1 Solanezumab-treated patient and 2 placebotreated patients [20]. Overall, it was concluded that treatment benefits and improvements were present with Solanezumab therapy, but the overall clinical impact in reducing cognition decline did not reach statistical significance. The primary endpoint was not met, and the secondary analyses of the previous 2 expeditions were not reproduced.

Another phase 3 study, ExpeditionPro, was initiated to assess whether Solanezumab can aid patients with a clinical diagnosis of prodromal AD instead [21]. The dosing regimen consisted of an intravenous dose administered once per month over a 2-year course [21]. However, the trial was terminated due to unsatisfactory scientific evidence in favor of a meaningful benefit. Solanezumab did not meet the primary endpoint in obtaining a statistically significant slowing in cognitive decline; an 11 percent decrease in decline was measured by ADAS-Cog14 ( $p=$ 0.095) [22].

Consequently, Eli Lilly \& Co. discontinued their pursuit toward FDA approval of Solanezumab. This failure resulted from a lack of translation of the antibody's strong target engagement - as indicated by the elevated plasma $A \beta 40$ and $A \beta 42$ levels - into a clinically relevant response. Specifically, its biological efficacy against brain $A \beta$ plaques was insufficient to cause an improvement in patient cognition. Nevertheless, the treatment effects were consistent across the various clinical measures, as demonstrated by the separated curves between Solanezumab and placebo. Given that the role of Solanezumab is to increase the $\mathrm{A} \beta$ clearance rate, patients with higher levels of $A \beta$ at baseline would obtain a larger clinical benefit. Thus, in the future, screening methods can be applied to ensure that the baseline $A \beta$ level becomes an additional inclusion criterion [23].

\section{Aducanumab (BI-IB037): Amyloid Remover}

(Anti-A $\beta$, Targets Oligomers and Plaques, and

Disease-Modifying Treatment)

Aducanumab is a human monoclonal IgG1 antibody that selectively targets aggregated forms of $A \beta$, such as soluble oligomers and insoluble fibrils, but excludes $A \beta$ monomers. After crossing the blood-brain barrier, the antibody engages with its target in the CNS, showing preference for parenchymal over vascular A $\beta$ [24]. Specifically, it binds to a conformational epitope located at the $\mathrm{A} \beta \mathrm{N}$-terminus [24]. Consequently, CNS A $\beta$ will be cleared from the plaque-bearing brain.

Originally discovered by Neurimmune, this drug was derived from a library of $\mathrm{B}$ cells gathered from healthy aged individuals. The researchers reasoned that the operative antibodies generated from these donors can be converted into therapeutics - using the reverse translational medicine method - since their immune systems have already successfully opposed the development of $\mathrm{AD}$ [24]. Biogen Inc. then licensed the drug candidate from Neurimmune and further developed this antibodybased immunotherapeutic approach. Instead of pursuing healthy matured individuals, they screened for memory B-cell clones originating from elderly subjects who suffer from cognitive impairment but have slow cognitive decline [24]. Hence, the resulting activated antibodies would show reactivity against aggregated $A \beta$ since their release was triggered by the neo-epitopes present in the aggregates. Subsequently, this process led to the molecular cloning, sequencing, and recombinant expression of Aducanumab. The hypothesis was that Aducanumab would reduce the level of brain $A \beta$ plaques, which, in turn, will elicit clinical benefit.

Given the successful target engagement and clinical benefits it provided in Prime, a double-blind randomized phase $1 \mathrm{~b}$ study, Aducanumab was advanced to phase 3 clinical trials in September 2015. This stage consisted of Engage and Emerge, 2 identically designed, parallel-group studies conducted under double-blind, randomized, and placebo-controlled conditions $[25,26]$. The inclusion criteria were $\mathrm{AD}$-associated mild cognitive impairment or mild AD dementia, along with the following: MMSE score within 24-30, CDR-G score of 0.5, Repeatable Battery for the Assessment of Neuropsychological Status (RBANS) score of $\leq 85$, and a positive A $\beta$ PET scan [25, 26]. Notably, approximately two-thirds of the participants are carriers of the APOE $\varepsilon 4$ allele, the strongest genetic risk factor for late-onset $\mathrm{AD}[27,28]$. Its disproportionate frequency of about $36.7 \%$ in $\mathrm{AD}$ patients exists at an average frequency of $\sim 13.7 \%$ worldwide $[27,28]$. For the dosing regimen, Aducanumab was administered every 4 weeks at either low or high doses for 18 months. Particularly, the low dose contained $3 \mathrm{mg} / \mathrm{kg}$ for the APOE $\varepsilon 4$ carriers and $6 \mathrm{mg} / \mathrm{kg}$ for noncarriers; the high dose contained 6 and $10 \mathrm{mg} / \mathrm{kg}$, respectively [29]. A lower drug dose was given to individuals who are APOE $\varepsilon 4$ carriers due to ARIA-E susceptibly.

After 1.5 years of treatment, protocol version 4 (PV4) was implemented in March 2017. This involved titration of the high dose APOE $\varepsilon 4$ carrier group's original drug 
level from 6 to $10 \mathrm{mg} / \mathrm{kg}$ [30]. The primary endpoint was to assess cognitive and functional decline with CDR-SB. The secondary outcomes included measurements of MMSE, ADAS- $\operatorname{cog} 13$, and ADCS-ADL-MCI scores. The sub-studies involved analysis of amyloid PET, tau PET, and CSF biomarkers [31].

An interim futility analysis was performed in December 2018 on 945 Engage and 803 Emerge patients to predict whether the 2 trials would meet their primary endpoint. The results showed that the Emerge data were trending positive, but those of Engage were, unfortunately, trending negative [29]. With this discrepancy, Biogen estimated failure to obtain statistical significance at the planned final analysis, which led them and their collaborator Eisai to officially end these trials [32].

During the 3-month period between the start of the futility test and the subsequent termination of the trials, additional 139 and 179 individuals had completed Engage and Emerge, respectively [32]. When these results were added to generate a larger combined dataset, a reassessment had led to the realization that their previous analysis had been incorrect [32]. Precisely, the conclusions made were based on the data available as of December 2018, which meant that only patients who had already completed the 18-month program by that time were included [33]. These individuals had also received lower average drug exposure than those enrolled later, due to the nature of the dosing regimen [33]. As a result, their cumulative dosage level may not have been sufficient to provide a reduction in clinical decline [33]. Thus, the additional data collected within these 3 months permitted the inclusion of patients that were able to acquire sufficient exposure to high-dose Aducanumab due to their later enrollment date.

In turn, this led to statistically significant results for the high-dose Emerge dataset - but not for the high-dose Engage dataset. This is justified as the Emerge trial started 1 month later than Engage, which resulted in more patients receiving the higher dose as less participants were enrolled at the time of the 2017 PV4 amendment [32]. For high-dose Emerge, a dose-responsive effect was observed in the CDR-SB scale, where a $23 \%$ improvement had occurred $(p=0.031)$ [32]. However, the effects on its secondary endpoints were lower. For high-dose Engage, the results still did not demonstrate a reduction in clinical decline; a $6 \%$ worsening in performance on the CDR-SB scale was measured ( $p=0.627$ ) [32]. Though, a dose-responsive enhancement was seen for the ADAS-Cog13 and ADAS-ADL-MCI scores. Similar results were observed for the larger Emerge (1,638 patients) and Engage $(1,647$ patients) dataset: $23 \%$ improvement $(p=0.010)$ and $2 \%$ worsening ( $p=0.825$ ) for CDR-SB, respectively [32]. All results from the Engage and Emerge trials as part of the larger dataset were released at the 12th Clinical Trials on Alzheimer Disease (CTAD) conference.

For the Engage study, an original set of 1,350 patients with early stage $\mathrm{AD}$ were recruited, which was later increased to 1,647 for the purpose of preserving the statistical power of the results. The Emerge study was conducted with identical conditions as Engage, which also originally recruited 1,350 patients and was then increased to a total of 1,643. Despite the negative findings for Engage, its post hoc analysis revealed that data from a subset of its highdose patients provided support for the positive Emerge results (116 and 147 patients, respectively) [32]. Both subsets were administered with more than 10 uninterrupted $10 \mathrm{mg} / \mathrm{kg}$ dosing intervals at steady state [31]. It was found that the Engage patients experienced a slow decline similar to comparable Emerge subjects: -0.45 and -0.75 points change in CDR-SB relative to placebo $[25,26]$. Secondary endpoints followed a similar pattern [31].

An unanswered question is whether the Emerge results are positive and well-controlled [32]. Emerge's reported attainment of a $23 \%$ relative improvement on the CDRSB scale translates to an absolute difference of 0.4 CDR$\mathrm{SB}$ points, which is of ambiguous clinical relevance [32]. It was argued that this treatment effect may be overemphasized since the sample size was smaller than originally intended; $40 \%$ of Emerge patients had discontinued the trial [32]. If the sample size was as large as planned, the result might have regressed towards the mean. Moreover, it was stated that the improvement could have been a consequence of the placebo group experiencing greater deterioration as opposed to the experimental group gaining benefits from receiving greater exposure to high-dose Aducanumab. Other limitations that may have impacted the validity of Emerge's results include the following: its elevated dropout rate; greater absent doses; decreased treatment compliance; and unblinding of the high-dose regimen due to the presence of ARIA-E in APOE $\varepsilon 4$ carriers, who made up two-thirds of the total randomized participants, as compared to the low dose and placebo groups [32]. Furthermore, the patients selected to be apart from the Emerge high-dose subset consisted of just $20 \%$ of the overall participants in the high-dose group. This subgroup included individuals who completed the full trial, were compliant with the treatment plan, and were mostly APOE $\varepsilon 4$ noncarriers with lower ARIA vulnerability. Consequently, when these patients were assessed against the randomized placebo group, any comparison made may have been biased since those noncar- 
riers would have a smaller decline than the placebo APOE $\varepsilon 4$ carriers [32].

Overall, the dosage level of Aducanumab was found to be important for its efficacy towards reducing clinical decline in AD. The drug was able to decrease brain $A \beta$ and CSF phospho-tau levels.

Accordingly, Biogen will be seeking FDA approval for Aducanumab in 2020, which, if successful, will be the first new AD therapy in over a decade [29]. In January 2020, Biogen conducted a Phase $3 \mathrm{~b}$ open-label trial for 2,400 Aducanumab participants, which will involve administration of $10 \mathrm{mg} / \mathrm{kg} /$ month injections for 2 years [31]. Safety and tolerability parameters are the primary endpoints, and the expected end date is September 2023 [31].

\section{Crenezumab (MABT5102A, RG7412): Amyloid}

Remover (Anti-A $\beta$, Targets Oligomers, and

Disease-Modifying Treatment)

Genentech and Roche's Crenezumab are a humanized monoclonal IgG4 antibody that effectively binds to multiple forms of aggregated $A \beta$ with high affinity: that is, oligomers, fibrils, and plaques [34]. Within this broad binding profile, it has a 10 -fold higher affinity towards soluble oligomers [27]. Some evidence supports that soluble oligomers are primary drivers of $\mathrm{A} \beta$-related neurotoxicity [27]. Such conformational specificity towards the relevant $A \beta$ species increases its overall efficacy in exerting a meaningful biological effect since off targets, such as A $\beta$ monomers, are excluded. Consequently, when Crenezumab binds to oligomers at the periphery of plaques, oligomer-induced neurotoxicity becomes inhibited [29].

Like Solanezumab, Crenezumab targets $A \beta$ at its midregion. Specifically, it does so at amino acids $16-24$ and functions to clear excess $A \beta$. It can also block $A \beta$ aggregation and stimulate $A \beta$ disaggregation of oligomers [35]. Its IgG4 backbone, unlike that of IgG1, confers the advantage of having reduced activation of $\mathrm{Fc}$-gamma receptors (Fcy6Rs) on CNS macrophages [35]. This decreases their subsequent effector function, preventing inflammation as the release of cytokines and other mediators become diminished - which would otherwise trigger neurotoxicity [35]. However, Fc $\gamma \mathrm{R}$-mediated microglial phagocytosis of oligomers still remains viable as a way for $A \beta$ clearance $[7,35]$. As such, use of the IgG4 backbone will circumvent the undesirable vascular side effects that are associated with IgG1-designed antibodies. These include ARIA-E, ARIA-H, and complement-dependent cytotoxicity $[7,36]$. Hence, with a lower risk of inducing ARIAs, patient safety is increased and potential limitations to drug dosage levels can be alleviated.

Anti-Amyloid Monoclonal Antibodies in Clinical Trials
Advanced to phase 3 clinical trials, 750 prodromal-tomild AD patients were recruited for the Cread study. The safety and efficacy of the $60 \mathrm{mg} / \mathrm{kg}$ dose was examined to see whether treatment benefits can be obtained without the associated safety findings described for previous anti$A \beta$ immunotherapies [29]. Its primary outcomes involved assessing CDR-SB changes whereas its secondary outcomes focused on cognitive and functional measures: ADAS-Cog and MMSE [34]. However, the results were exclusively negative. No difference was found for both endpoints - primary and secondary - between the treatment versus placebo groups or within the prodromal versus mild AD subgroups [34]. A Cread2 study of 750 prodromal-to-mild AD members was also initiated [34]. Though, this trial was terminated alongside Cread - also due to failure to obtain a significant drug-placebo difference [34]. Despite target engagement, Crenezumab had no efficacy in treating AD symptoms. However, Solanezumab, which is similar in its binding to Crenezumab, elicited real - although insignificant - treatment benefits. Perhaps, it is too late for even patients at the earlier stages of $\mathrm{AD}$, whose brain already consists of mature plaques, as well as significant tau pathology and neuroinflammation to respond Crenezumab's inhibitory effect on A $\beta$ aggregation.

\section{Gantenerumab (RO4909832, RG14502): Amyloid}

Remover (Anti-A $\beta$, Targets $A \beta$ Fibrils, and

Disease-Modifying Treatment)

Roche's Gantenerumab is a monoclonal IgG1 antibody that targets $A \beta$ fibrils with subnanomolar affinity [37]. It binds at a conformational epitope that encompasses the $\mathrm{N}$-terminal and central amino acids in a configuration that cannot be achieved with the structure of A $\beta$ monomers [38]. Specifically, peptides 8 (AEFRHDSGYE) and 9 (EFRHDSGYEV) are targeted at the N-terminus while peptide 24 (VFFAEDVGSN) is targeted within the central section of the A $\beta$ fibril [38]. Unlike Solanezumab, which is a humanized version of a murine antibody, Gantenerumab is the first fully human anti-A $\beta$ monoclonal antibody to begin clinical development [13]. The human aspect of this antibody sets it apart from the other xenogenic antibodies, as it provides greater suitability for chronic administration. Its development involved the use of phage display technology to screen and select for drug candidates from the synthetic human combinatory antibody library [13]. Optimization was then performed via in vitro affinity maturation on $A \beta$ fibrils to obtain the highest target affinity and specificity [13]. In human clinical trials, initially set up as a phase 2 study

Dement Geriatr Cogn Disord 2020;49:334-348 339 
involving 360 participants, it was later expanded into a phase $2 / 3$ study called the SCarlet RoAD trial that currently assesses the efficacy and safety of Gantenerumab in 799 prodromal AD patients [39]. In part 1 of the study, Gantenerumab was administered at either 105 or 225 $\mathrm{mg} / 4$ weeks via subcutaneous injections in the abdomen for a total of 104 weeks [39]. The primary endpoints were to measure changes in the CDR-SB score at week 104 and brain $A \beta$ levels while the secondary endpoints included changes in cognition, behavior, and daily function [39]. Particularly, cognition was evaluated using ADAS-Cog 13, the MMSE, the Cambridge Neuropsychological Test Automated Battery (CANTAB), and the Free and Cued Selective Reminding Test (FCSRT) [39]. Behavior was evaluated with the NPI Questionnaire (NPI-Q), and daily functioning was evaluated through the Functional Activities Questionnaire (FAQ) [39]. After completion of the 104-week period, the option to continue treatment for an additional 2 years was offered, as part 2 of the study. When at least $50 \%$ of the patients had finished 2 full years of treatment, a futility interim analysis was conducted. From this, it was concluded that the probability of obtaining efficacy on the primary and secondary endpoints in the SCarlet RoAD trial was low, which led to an early termination [39].

However, post hoc analysis revealed that clinical benefit was present with the fastest progressors; these are patients whose CDR-SB performance and hippocampal volume had reduced the greatest throughout the duration of the trial [37]. Particularly, a concentration-dependent effect on ADAS-Cog and MMSE was achieved [40]. Additional analysis also showed biomarker and efficacy signals among the high-dose recipients in this group [37]. Based on this finding, the focus was shifted toward open label to further test the effects of injecting higher doses of Gantenerumab (part 3 of the study), which will run until July 2020 [37]. The altered regimen involves injecting doses up to $1,200 \mathrm{mg} / 4$ weeks for 3 additional years [37]. In terms of safety, the percentage of individuals experiencing adverse events or serious adverse events will be measured. Thus far, it was found that lower amounts of ARIAE are experienced with slower titration speeds [37].

Despite the failing results of the interim analysis, Roche initiated another phase 3 trial called Marguerite RoAD. It was designed to evaluate Gantenerumab at the same dosage level as that of $S$ Carlet RoAD, but mild AD - in a total of 389 participants - was targeted instead of prodromal $\mathrm{AD}$ [41]. The aim was to evaluate the efficacy and safety of Gantenerumab. However, like SCarlet RoAD, Marguerite RoAD also failed its interim futility analysis, causing a halt in the usage of the low dose. Consequently, part 2 of the study was initiated as an openlabel extension study, where patients are titrated up to 1,200 mg of Gantenerumab [37]. The co-primary endpoints are ADAS-Cog and ADCD-ADL while the biomarkers, clinical, and neuropsychiatric measures are the secondary endpoints. The trial is still active and has an anticipated completion date set for April 2021 (Tables 1, 2).

From the SCarlet RoAD and Marguerite RoAD trials, 81 patients were enrolled into open-label extension PET sub-studies; 40 individuals - 14 in Marguerite RoAD-Placebo, 17 in Marguerite RoAD-Gantenerumab, and 9 in SCarlet RoAD - had met the standards for high-dose analysis [42]. Changes in $\mathrm{A} \beta$ burden were measured via acquiring $\left[{ }^{18} \mathrm{~F}\right]$ Florbetapir PET scans at baseline and week 52. The results showed that the magnitude of benefit tripled that of the 2-year change observed with the 225 $\mathrm{mg}$ dose from the SCarlet RoAD trial [42]. Specifically, the mean change observed was -0.24 (0.21) for Marguerite RoAD-Placebo, - 0.27 (0.14) for Marguerite RoAD-Gantenerumab, and $-0.13(0.16)$ for SCarlet RoAD in SUVR units [42]. By the end of the treatment, the $A \beta$ levels in a third of the participants had dropped below the threshold for $A \beta$ positivity [42].

Moreover, in 2012, a phase 2 study was performed to assess the safety, tolerability, and biomarker efficacy of Gantenerumab versus Solanezumab in patients at risk for or with the rare autosomal dominant Alzheimer disease (ADAD) gene mutation. Accounting for $<1 \%$ of all cases, it is specifically an early onset inherited form of $\mathrm{AD}$ [43]. This trial involved a total of 210 participants, where either a subcutaneous dose of Gantenerumab at $225 \mathrm{mg} / 4$ weeks $(n=52)$ or an intravenous dose of Solanezumab at 400 $\mathrm{mg} / 4$ weeks $(n=50)$ was administered [13]. Changes in the amount of $A \beta$ plaque deposition were measured by taking $\left[{ }^{11} \mathrm{C}\right]$-PiB-PET scans at baseline and 2-year posttreatment to determine biomarker target engagement [44]. Afterward, cognitive efficacy was examined over a 7-year follow-up period, as part of phase 3 [45]. Precisely, the primary outcome involved changes in the Dominantly Inherited Alzheimer Network Trials Unit (DIAN-TU) score [44]. The results revealed that both drugs failed to significantly slow the rate of cognitive decline. By year 4 , neither of the treatment cohorts performed better than placebo [45]. Though, it should be noted that the study began with low doses and was then titrated four- and fivefold higher only midway through the trial for Solanezumab (September 2017) and Gantenerumab (August 2016), respectively $[44,45]$. Consequently, the high dose may 


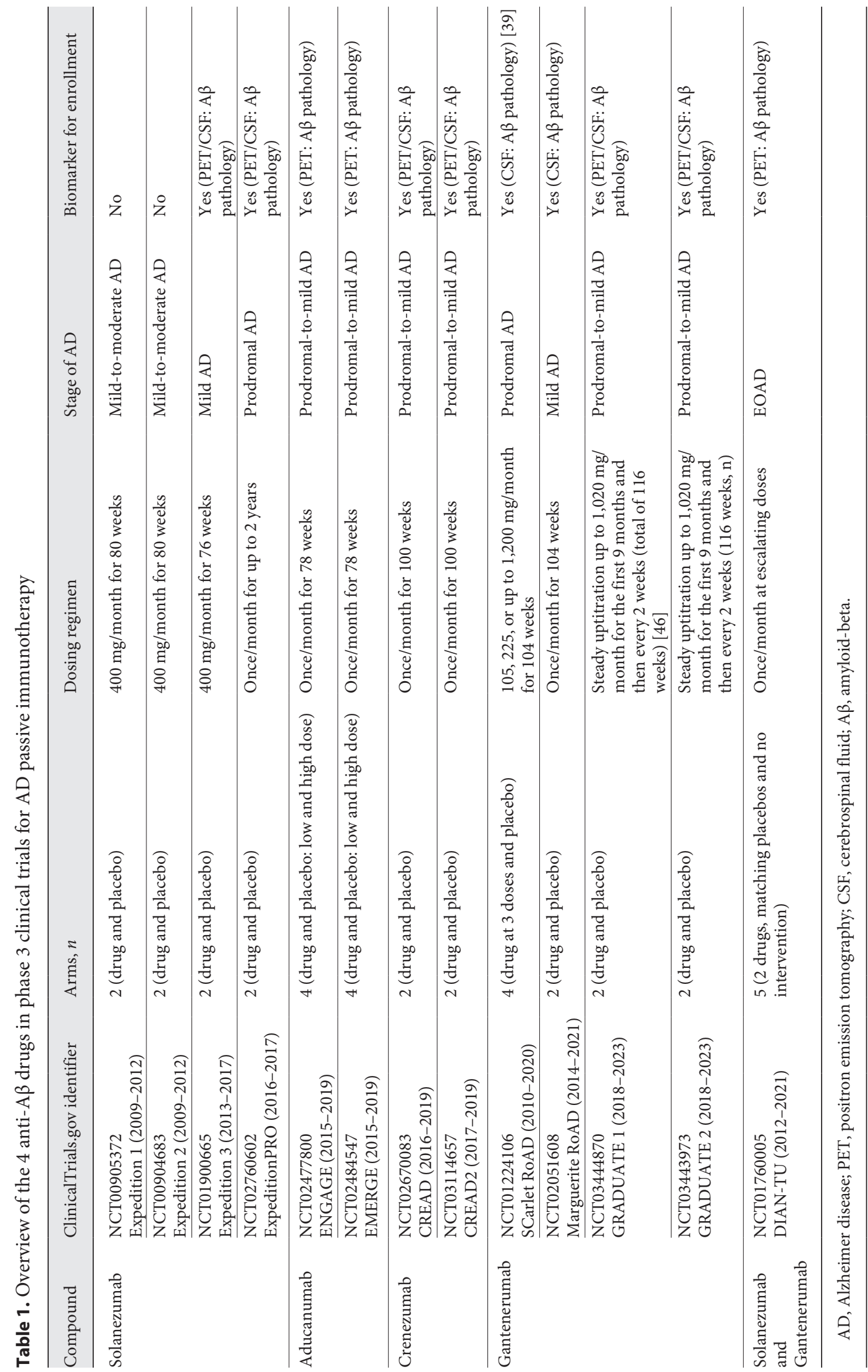




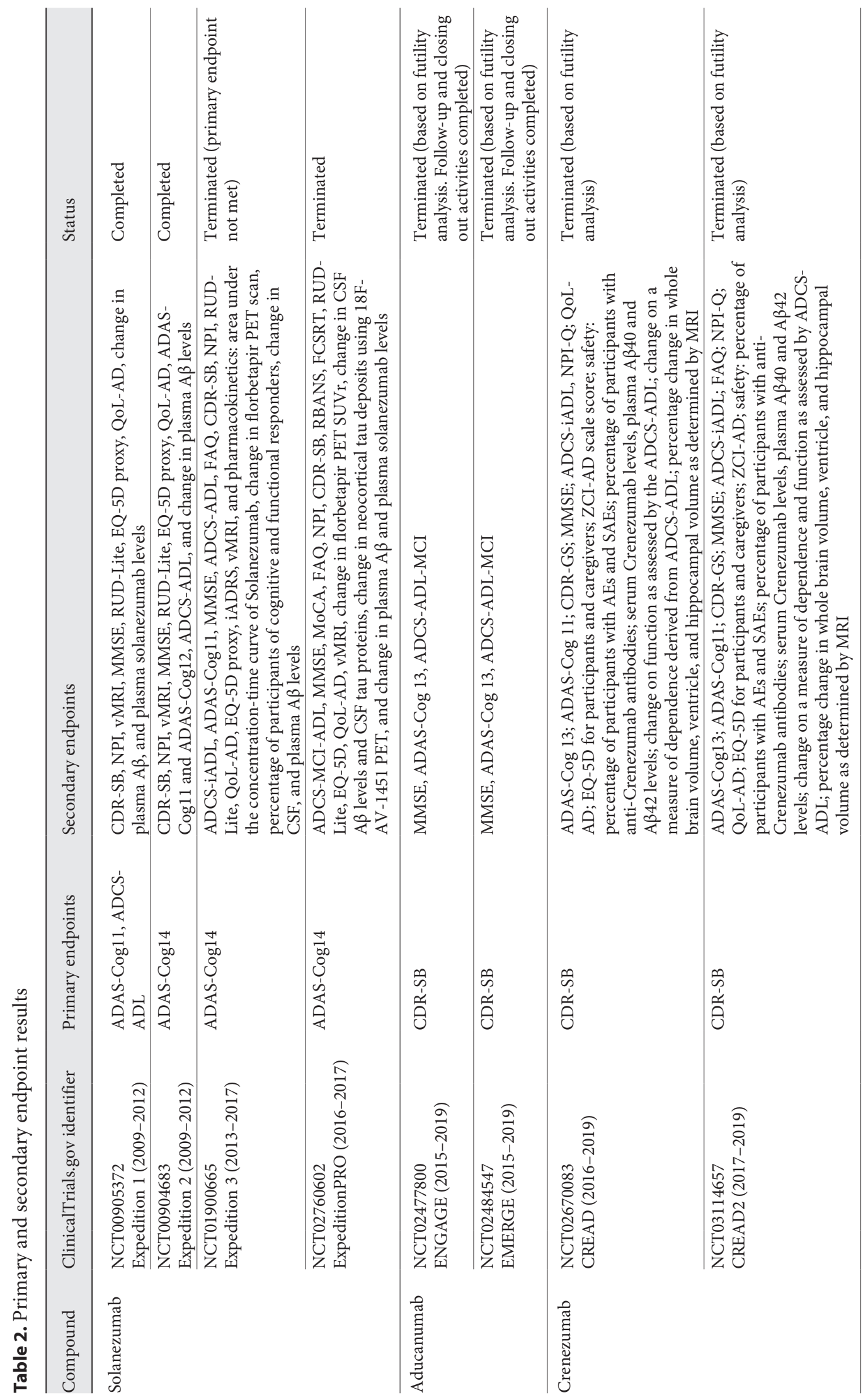




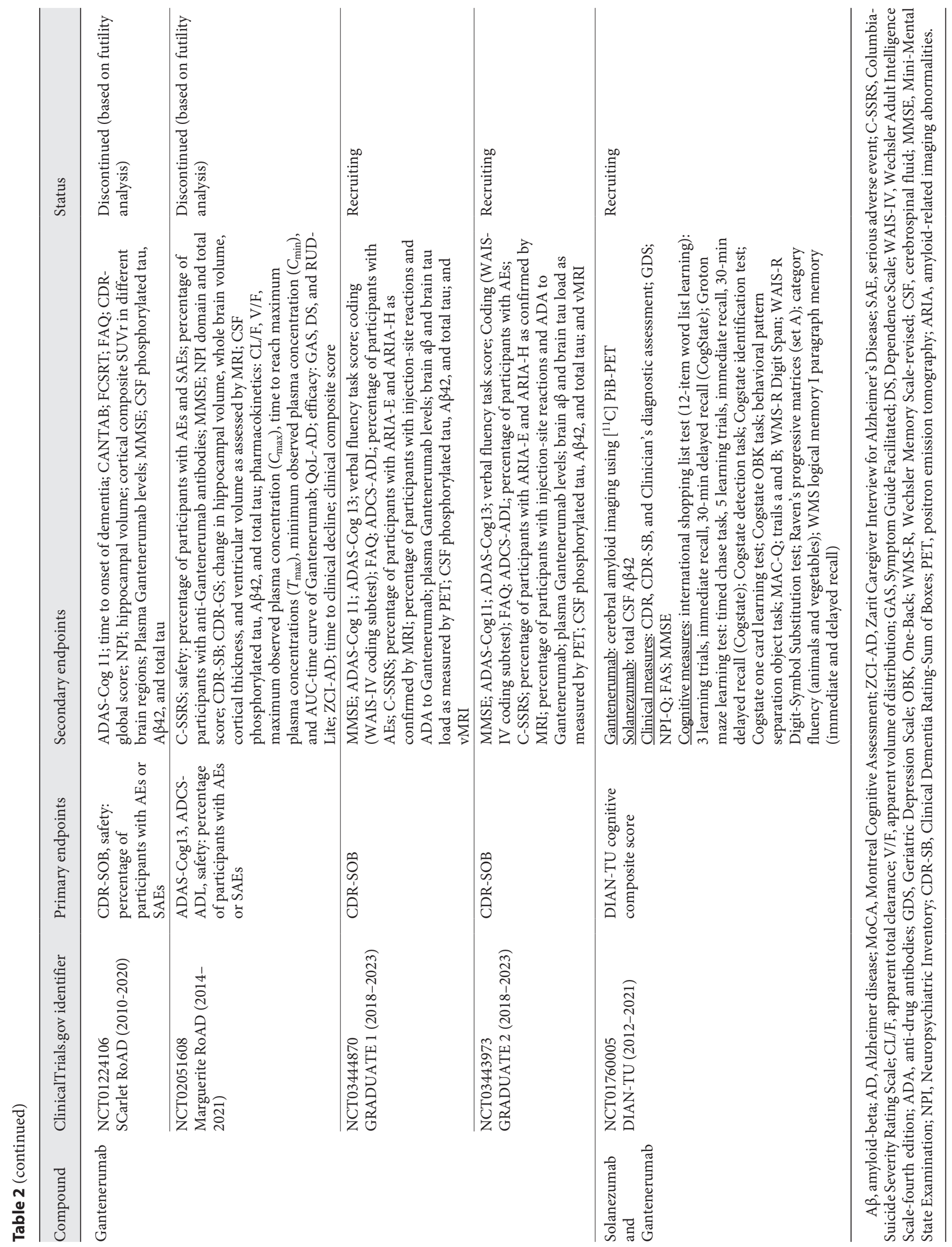


not have been tested for long enough, potentially causing underdosage of the study. The titration also came late, as symptomatic patients already declined significantly and developed moderate dementia before being treated with the high dose. The asymptomatic patients remained stable despite receiving drug or placebo [45]. Additionally, participants experienced adverse events such as injection-site reactions, nasopharyngitis, and ARIA that developed into cerebral edema or microhemorrhages. Overall, these findings signified an end for further investigation in DIAN-TU for Solanezumab, but not for Gantenerumab [45]. Alongside its ability to eliminate cerebral A $\beta$ plaques and normalize CSF A $\beta 42$, Gantenerumab had also normalized elevated total CSF tau and ptau181 levels and inhibited increases in CSF neurofilament light (NfL), a neurodegeneration marker [45]. A $\beta$ was, thus, proposed to be responsible for triggering the significant tau and NfL downstream changes [45]. Subsequently, the DIAN investigators initiated a 3-year openlabel extension, DIAN-TU-001, to explore the effects of receiving sustained Gantenerumab therapy at the highest tolerated dose for several more years. Currently, the dose for mutation carriers has been set at 1,020 mg per infusion, which may increase further should ARIA and tolerability permit [45]. The purpose was to determine whether the amount of $A \beta$ plaques and tangles could be reduced to a threshold that permitted clinical benefit in cognition and function [45]. With clearance of pathology at doses that are well tolerated, the next step would be to assess if the brain can heal from the significant neurodegeneration seen in $\mathrm{AD}$ through dampening its inflammation and restoring synapses and circuits [45].

At the AAT-AD/PD 2020 virtual conference, the data analysis of the presymptomatic group was examined separately from those who were symptomatic, as measured at baseline. The former improved on both the logical memory and Digit-Symbol Substitution (DSS) tests while the latter failed to improve and declined, respectively [45]. On the MMSE, presymptomatic individuals remained stable near 30 whereas symptomatic individuals decreased from 26 to 20 [45]. This trend is applied to CDR-SB and the functional assessment scale (FAS) as well [45]. In general, presymptomatic participants began closer to the tests' normal range and remained stable; symptomatic participants started off worse and declined [45]. Therefore, the lack of evidence supporting the cognitive endpoint could have resulted from subtle changes in cognition and function that went undetected [45]. Another source of the problem stems from the dominantly inherited $\mathrm{AD}$ (DIAD) disease-progression model, which provides the statistical construct to analyze the primary endpoint data in the 2 trial arms [45]. Specifically, the model made assumptions that the trial data did not meet: that is, all test performances would decline, variance would be constant across presymptomatic and symptomatic patients, and about when symptoms would initiate [45]. However, it was found that the placebo group improved on certain tests, asymptomatic and symptomatic carriers had different degrees of variance on the MMSE, and measures did not change longitudinally in the manner outlined by cross-sectional analysis [45]. In terms of safety, the side effects measured in the DIAN-TU-001 trial were similar to previous trials. No ARIAs were caused by Solanezumab and Gantenerumab demonstrating great tolerability.

The biomarker analysis was then presented. For Solanezumab, a large increase in total CSF A $\beta 42$ levels signified successful target engagement. The CSF total tau and p-tau 181 remained unchanged. Only results from CSF NfL of the downstream markers were different relative to placebo, though, the change represented worsening ( $p=$ 0.017) [45]. Moreover, FDG PET and precuneus thickness - downstream markers of neurodegeneration - were not different in the combined asymptomatic/symptomatic cohort with Solanezumab or Gantenerumab. For Gantenerumab, target engagement was shown via its $\left[{ }^{11} \mathrm{C}\right]-\mathrm{PiB}-\mathrm{PET}$ results, where a significant reduction was obtained at 0.64 SUVR [45]. When the cohort was analyzed separately for the presymptomatic and symptomatic groups, it was observed the presymptomatic and symptomatic groups had different levels of $\left[{ }^{11} \mathrm{C}\right]-\mathrm{PiB}-$ PET centiloids at baseline and follow-up. Presymptomatic carriers began near 30 centiloids, in which placebo recipients gained 10 centiloids over the course of the trial, and Gantenerumab patients lost 10 centiloids over the course of the trial. Symptomatic carriers began near 85 centiloids, where placebo recipients exceeded 100 centiloids by the end of the trial and Gantenerumab recipients reduced to 65 centiloids by the end of the trial. Mutation noncarriers reported zero centiloids throughout the trial [45]. Based on this data, it was concluded that a large baseline-difference was observed in the 2 groups.

The reduced $A \beta$ load, though, was not associated with cognitive benefit; it is hypothesized that a decrease in 30 40 centiloids would be required [45]. The CSF A $\beta 42 / 40$ ratio increased while the CSF total tau and p-tau 181 decreased by a third, all of which signified a biologically significant reversal toward normal levels $(p<0.001)$ [45]. By year 4 , both tau measures showed at least a $30 \%$ difference relative to placebo; levels in placebo recipients worsened 
while those in noncarriers remained unchanged [45]. Its CSF NfL levels, however, exhibited a smaller increase relative to placebo $(p=0.024)$ but stayed stable in noncarriers [45]. With regard to A $\beta$ burden, a $209 \%$ reversal toward normal levels was measured by year 4 . Overall, this OLE will allow researchers to determine if high-dose Gantenerumab can decrease $\mathrm{A} \beta$ plaques in DIAD individuals below the threshold of positivity. If this is attained, then whether downstream markers of $\mathrm{AD}$ will return to normal must be addressed as well. Last, clinical benefit will be measured to see if symptomatic carriers can stabilize or improve, and presymptomatic individuals continue to remain well. The OLE is set to start once the situation for COVID19 permits.

In the phase 3 Graduate clinical program, Gantenerumab is being investigated in 2 trials named $\mathrm{Gradu}$ ate 1 and Graduate 2 to further test whether a higher dose will lead to clinical benefit regarding $A \beta$ reduction. Participants who have early, sporadic $\mathrm{AD}$ were registered with prodromal or mild $A D$ dementia, who are $A \beta$ positive - where the estimated enrollment is at 1,016 [37]. The regimen involves administering Gantenerumab as SC injections with steady uptitration up to $1,020 \mathrm{mg}$ for a total of 104 weeks [46]. The primary outcome is to measure the change in the CDR-SOB score, after which entrance to an OLE will be offered [37]. Otherwise, there will be a longterm follow-up period for 50 weeks. Currently, both trials are active and still recruiting. The predicted study completion date is set at May 2023.

\section{Discussion}

Finding a viable treatment for $\mathrm{AD}$ has proven challenging. Over the past 15 years, nearly all pharmaceutical therapies have failed at the clinical development stage, which has raised many questions about the future of $\mathrm{AD}$ drugs [47]. During this time, the consensus established among researchers has stressed the importance of the $A \beta$ cascade pathway in being the primary driver of $\mathrm{AD}$ pathogenesis. However, the resulting amyloid-targeting therapeutics, and particularly DMTs, that function to reduce A $\beta$ levels, have either failed or been discontinued $[11,48]$.

While the failure of anti-amyloid therapies to meet primary endpoints is discouraging, many questions should be addressed before these trials can be used as evidence against the amyloid cascade hypothesis of AD. Accepted disease models from $\mathrm{ADAD}$ and sporadic $\mathrm{AD}$ suggest amyloid accumulation begins decades prior to symptom onset $[49,50]$. In this disease model, a direct

Anti-Amyloid Monoclonal Antibodies in Clinical Trials relationship between cerebral amyloid levels and cognitive decline trajectories is not expected. Moreover, several early anti-amyloid therapies recruited individuals based on clinical criteria which have limited sensitivity and specificity for AD pathology [51-54]. This may have resulted in using anti-amyloid therapies on individuals who did not harbor amyloid pathology. For instance, early studies did not monitor target engagement, thus limiting conclusions about the efficacy amyloid reduction in AD. Finally, many trials were conducted in individuals with advanced disease (dementia), characterized by widespread cortical neurodegeneration, where the $\mathrm{AD}$ process may have been far too advanced to be halted by anti-amyloid mechanisms. Therefore, before rejecting $A \beta$ as a therapeutic target in $\mathrm{AD}$, future trials should focus on preventing cognitive decline in cognitively unimpaired individuals with evidence of cerebral amyloidosis.

When assessing the efficacy of anti-amyloid drugs in the treatment of Alzheimer's disease, it is necessary to consider the following: (1) are the subjects chosen for AD treatment appropriate (i.e., do the subjects have biomarker evidence of $A D$ pathological changes)? (2) what sample size would be most appropriate? (3) when would we expect anti-amyloid therapeutics to be effective in $\mathrm{AD}$ ? and (4) what drug dosage is optimal for treatment? The question of treating the right dementia patients is far from trivial. It is imperative that clinical trials adhere to inclusion criteria where amyloid PET scans are obtained for all patients at the initial recruitment phase, such that amyloid elevation levels can be accurately evaluated throughout the studies [55]. Otherwise, inappropriate patient recruitment would add noise and contribute to negative results. Additionally, a balance must be maintained between the sample size, its anticipated effect size of the intervention, and time and cost feasibility of the trial. Addressing the third question is dependent on understanding the sequences of progressive brain pathology. Recently, the biomarker model of $\mathrm{AD}$ shows that this process begins with amyloid buildup, then metabolic decline, followed by CSF tau accumulation and eventually brain volume reduction [56]. Since cognitive impairment is the last event in this sequence, it is very likely that most DMTs were administered far too late $[14,57]$. Finally, optimal drug dosage is essential to the success of clinical trials since late increases in dosage may negatively impact trial results [44].

There are known differences between ApoE genotypes in the risk of ARIA, the $4 / 4$ carriers being more at risk compared to $4 / 3$ or $3 / 3$, likely because of their higher amount of amyloid deposition in the media of blood ves- 
sels [58-61]. Some study protocols have been amended to lower doses of monoclonal antibodies in $4 / 4$. There has been to our knowledge no specific hypothesis in the phase 3 studies reviewed in this document as to the expected differences in response to treatment based on ApoE genotype. This situation will likely change in future phase 3 studies, considering the strength of data from Alzheon demonstrating that $4 / 4$ carriers greatly improved using the oral anti-oligomer binding agent ALZ-801, currently in phase 3 only for $4 / 4$ symptomatic subjects [58-61].

An immediate alternative to amyloid reducing therapeutics would be those targeting tau. These include taucentric active and passive immunotherapies, microtubule-stabilizing agents, tau protein kinase inhibitors, and tau aggregation inhibitors [60]. Drug candidates that inhibit the development of tau oligomers and fibrils have arrived at the clinical trial stage.

\section{Future Directions}

Researchers are now exploring drug approaches that target amyloidogenic pathways in more than 1 place [9]. Hence, effective therapies could focus on targeting either a key pathway at multiple timepoints or 2 or more pathways simultaneously. Known as combinational therapy, this approach involves administering 2 or more compounds, either sequentially or simultaneously, to slow or stop disease progression [9]. Future directions merit investigating the additive, synergistic benefits focusing on anti-A $\beta$ and anti-tau drug combinations. Given that in $\mathrm{AD}$, neurodegeneration is characterized by senile plaques and hyperphosphorylated tau aggregations; the next logical steps would be to explore DMTs that halt their progression together [61]. Other avenues include using PET in preclinical or prodromal populations to select patients for biomarker-based clinical trial participation [62-64].
While the road to the development of effective AD therapeutics may be long, learning from the current clinical trials proves fruitful in devising better designs in the future and helping dementia patients. We hope that this detailed review of the clinical trial program of passive immunization drugs for $\mathrm{AD}$ will prove useful to the design of future clinical trial programs.

"Published in Celebration of the 30th Anniversary of the inception of Dementia and Geriatric Cognitive Disorders 1990-2020".

\section{Acknowledgement}

The authors would like to thank the Journal of Dementia and Cognitive Disorders for their ongoing support and polishing of the manuscript.

\section{Conflict of Interest Statement}

S.G. is a member of the scientific advisory boards of Biogen, Boehringer-Ingelheim, Lilly, and TauRx, is a member of the DSMB of ADCS, ATRI, and Banner-Health, and receives funding from CIHR, FQRS, and NIH. The other authors declare no conflicts of interest.

\section{Funding Sources}

No funding sources were received in the preparation of the manuscript.

\section{Author Contributions}

The original draft was written by A.T.H.K. and S.A.; J.T., P.R.N., and S.G. reviewed and edited the manuscript.

\section{References}

1 Jack CR, Bennett DA, Blennow K, Carrillo MC, Dunn B, Haeberlein SB, et al. NIA-AA research framework: toward a biological definition of Alzheimer's disease. Alzheimers Dement. 2018;14(4):535-62.

2 Jack CR, Therneau TM, Weigand SD, Wiste HJ, Knopman DS, Vemuri P, et al. Prevalence of biologically vs clinically defined Alzheimer spectrum entities using the National Institute on aging-Alzheimer's association research framework. JAMA Neurol. 2019;76(10): 1174-83.
3 Alzheimer's Association. 2019 Alzheimer's disease facts and figures. Alzheimers Dement. 2019;15(3):321-87.

4 Masters CL, Bateman R, Blennow K, Rowe CC, Sperling RA, Cummings JL. Alzheimer's disease. Nat Rev Dis Primers. 2015;1:1505618.
5 Alzheimer's Association. 2018 Alzheimer's disease facts and figures. Alzheimers Dement. 2018;14(3):367-429.

6 Robinson M, Lee BY, Hane FT. Recent progress in Alzheimer's disease research, part 2: genetics and epidemiology. J Alzheimers Dis. 2017;57(2):317-30.

7 Graham WV, Bonito-Oliva A, Sakmar TP. Update on Alzheimer's disease therapy and prevention strategies. Annu Rev Med. 2017; 68(1):413-30. 
8 Olivares D, Deshpande VK, Shi Y, Lahiri DK, Greig NH, Rogers JT, et al. N-Methyl D-Aspartate (NMDA) receptor antagonists and memantine treatment for Alzheimer's disease, vascular dementia and Parkinson's disease. Curr Alzheimer Res. 2013;9(6):746-58.

9 Cummings JL, Tong G, Ballard C. Treatment combinations for Alzheimer's disease: current and future pharmacotherapy options. J Alzheimers Dis. 2019;67(3):779-94.

10 Anderson RM, Hadjichrysanthou C, Evans S, Wong MM. Why do so many clinical trials of therapies for Alzheimer's disease fail? Lancet. 2017;390(10110):2327-9.

11 Huang LK, Chao SP, Hu CJ. Clinical trials of new drugs for Alzheimer disease. J Biomed Sci. 2020;27(1):18-3.

12 Vander Zanden CM, Chi EY. Passive immunotherapies targeting amyloid beta and tau oligomers in Alzheimer's disease. J Pharm Sci. 2020;109(1):68-73.

13 Novakovic D, Feligioni M, Scaccianoce S, Caruso A, Piccinin S, Schepisi C, et al. Profile of gantenerumab and its potential in the treatment of Alzheimer's disease. Drug Des Devel Ther. 2013;7:1359-64.

14 Van Dyck $\mathrm{CH}$. Anti-amyloid- $\beta$ monoclonal antibodies for Alzheimer's disease: pitfalls and promise. Biol Psychiatry. 2018;83(4): 311-9.

15 Karran E, Hardy J. A critique of the drug discovery and phase 3 clinical programs targeting the amyloid hypothesis for Alzheimer disease. Ann Neurol. 2014 Aug;76(2):185-205.

16 Imbimbo BP, Ottonello S, Frisardi V, Solfrizzi V, Greco A, Seripa D, et al. Solanezumab for the treatment of mild-to-moderate Alzheimer's disease. Expert Rev Clin Immunol. 2012;8(2):135-49.

17 GAN C, Hermans SJ, Parker MW, Miles LA Molecular basis for mid-region amyloid- $\beta$ capture by leading Alzheimer's disease immunotherapies. Sci Rep. 2015;5:2-6.

18 Zhang Y, Lee DH. Sink hypothesis and therapeutic strategies for attenuating Abeta levels. Neuroscientist. 2011;17(2):163-73.

19 Doody RS, Thomas RG, Farlow M, Iwatsubo T, Vellas B, Joffe S, et al. Phase 3 trials of solanezumab for mild-to-moderate Alzheimer's disease. N Engl J Med. 2014;370(4):311-21.

20 Honig LS, Vellas B, Woodward M, Boada M, Bullock R, Borrie M, et al. Trial of solanezumab for mild dementia due to Alzheimer's disease. N Engl J Med. 2018;378(4):321-30.

21 A Study of Solanezumab (LY2062430) in Participants With Prodromal Alzheimer's Disease (ExpeditionPRO) [Internet]. ClinicalTrials.gov. Eli Lilly and Company; 2019 [cited 2020Sep7]. Available from: https://www.clinicaltrials.gov/ct2/show/NCT02760602.

22 Li Puma DD, Piacentini R, Leone L, Gironi K, Marcocci ME, De Chiara G, et al. Lilly announces detailed results of solanezumab phase 3 EXPEDITION3 study at the clinical trials on Alzheimer's disease (CTAD) 2016 meeting. Stem Cells. 2019;37(11):1467-80.
23 Sperling RA, Rentz DM, Johnson KA, Karlawish J, Donohue M, Salmon DP, et al. The A4 study: stopping $\mathrm{AD}$ before symptoms begin? Sci Transl Med. 2014;6(228):4-6.

24 Sevigny J, Chiao P, Bussière T, Weinreb $\mathrm{PH}$, Williams L, Maier M, et al. The antibody aducanumab reduces $\mathrm{A} \beta$ plaques in Alzheimer's disease. Nat Publ Gr. 2016;537(7618):50-6.

25 221AD301 Phase 3 Study of Aducanumab (BIIB037) in Early Alzheimer's Disease (ENGAGE) [Internet]. ClinicalTrials.gov . Biogen; 2020 [cited 2020Sep7]. Available from: https://clinicaltrials.gov/ct2/show/ NCT02477800.

26 221AD302 Phase 3 Study of Aducanumab (BIIB037) in Early Alzheimer's Disease (EMERGE) [Internet]. ClinicalTrials.gov Biogen; 2020 [cited 2020Sep7]. Available from: https://clinicaltrials.gov/ct2/show/ NCT02484547.

27 Cummings JL, Cohen S, Van Dyck CH, Brody $\mathrm{M}$, Curtis C, Cho W, et al. ABBY: a phase 2 randomized trial of crenezumab in mild to moderate Alzheimer disease. Neurology. 2018;90(21):E1889-97.

28 Liu CC, Liu CC, Kanekiyo T, Xu H, Bu G. Apolipoprotein e and Alzheimer disease: risk, mechanisms and therapy. Nat Rev Neurol. 2013;9(2):106-18.

29 Lin H, Ostrowitzki S, Sink KM, Millar L, Warren F, Smith J, et al. Baseline characteristics from phase 3 trial of crenezumab prodromal to mild Alzheimer's disease (CREAD). Alzheimers Dement. 2018;14(7S_Part_4):P217.

30 Rogers MB. Exposure, exposure, exposure? At CTAD, aducanumab scientists make a case. Alzforum. 2020;1-13.

31 Therapeutics: Aducanumab [Internet]. Alzforum. Biogen, Neurimmune; 2020 [cited 2020Sep7]. Available from: https://www.alzforum.org/therapeutics/aducanumab.

32 Schneider L. A resurrection of aducanumab for Alzheimer's disease. Lancet Glob Heal. 2019;4422(19):9-10.

33 Biogen. Biogen plans regulatory filing for aducanumab in Alzheimer's disease based on new analysis of larger dataset from phase 3 studies. Press Release. 2019;2019:1-9.

34 Therapeutics: Crenezumab [Internet]. Alzforum. AC Immune SA, Genentech, Hoffmann-La Roche; 2019 [cited 2020Sep7]. Available from: https://www.alzforum.org/ therapeutics/crenezumab.

35 Ultsch M, Li B, Maurer T, Mathieu M, Adolfsson O, Muhs A, et al. Structure of crenezumab complex with $A \beta$ shows loss of $\beta$-hairpin. Sci Rep. 2016;6(May):39374-11.

36 Crehan H, Lemere CA. Anti-amyloid- $\beta$ immunotherapy for Alzheimer's disease. In: Developing therapeutics for Alzheimer's disease. Amsterdam, Netherlands: Elsevier Inc; 2016. p. 193-226.

37 Therapeutics: Gantenerumab [Internet]. Alzforum. Chugai Pharmaceutical Co., Ltd., Hoffmann-La Roche; 2020 [cited 2020Sep7]. Available from: https://www.alzforum.org/ therapeutics/gantenerumab.
38 Bohrmann B, Baumann K, Benz J, Gerber F, Huber W, Knoflach F, et al. Gantenerumab: a novel human anti- $\mathrm{A} \beta$ antibody demonstrates sustained cerebral amyloid- $\beta$ binding and elicits cell mediated removal of human amyloid- $\beta$. J Alzheimers Dis. 2012;28(1):49-69.

39 Ostrowitzki S, Lasser RA, Dorflinger E, Scheltens $\mathrm{P}, \mathrm{Barkhof}$ F, Nikolcheva T, et al. A phase III randomized trial of gantenerumab in prodromal Alzheimer's disease. Alzheimers Res Ther. 2017;9(1):95-15.

40 Aducanumab, Solanezumab, Gantenerumab Data Lift Crenezumab, As Well [Internet]. Alzforum. 2015 [cited 2020Sep7]. Available from: https://www.alzforum.org/news/conference-coverage/aducanumab-solanezumab-gantenerumab-data-lift-crenezumabwell.

41 Klein G, Delmar P, Voyle N, Rehal S, Hofmann C, Abi-Saab D, et al. Gantenerumab reduces amyloid- $\beta$ plaques in patients with prodromal to moderate Alzheimer's disease: a PET substudy interim analysis. Alzheimers Res Ther. 2019;11(1):1-12.

42 Klein G, Delmar P, Hofmann C, Adjelkovic M, Abi-Saab D, Milosavljevic-Ristic S, et al. Higher dose gantenerumab leads to significant reduction in amyloid plaque burden - results for the marguerite and scarlet road open label extension studies. Neurology. 2018; 90(15 Suppl):S2.005.

43 Bateman RJ, Aisen PS, De Strooper B, Fox NC, Lemere CA, Ringman JM, et al. Autosomaldominant Alzheimer's disease: a review and proposal for the prevention of Alzheimer's disease. Alzheimers Res Ther. 2011; 2(6):1-13.

44 Topline Result for First DIAN-TU Clinical Trial: Negative on Primary [Internet]. Alzforum. 2020 [cited 2020Sep7]. Available from: https://www.alzforum.org/news/researchnews/topline-result-first-dian-tu-clinical-trial-negative-primary.

45 Bateman R, Aschenbrenner A, Benzinger T, Clifford D, Cruchaga C, Fagan A, et al. Results of the DIAN-TU prevention trial of solanezumab and gantenerumab in dominantly inherited AD. 2020;2-5.

46 Efficacy and Safety Study of Gantenerumab in Participants With Early Alzheimer's Disease (AD) [Internet]. ClinicalTrials.gov. Hoffmann-La Roche; 2020 [cited 2020Sep7]. Available from: https://clinicaltrials.gov/ct2/ show/NCT03444870.

47 Yiannopoulou KG, Papageorgiou SG. Current and future treatments in Alzheimer disease: an update. J Cent Nerv Syst Dis. 2020;12: 1179573520907397.

48 Hurley D. Why Alzheimer's investigators are undeterred by negative top-line results from DIAN-TU. Neurology Today. 2020;20(6):1.

49 Bateman RJ, Xiong C, Benzinger TL, Fagan AM, Goate A, Fox NC, et al. Clinical and biomarker changes in dominantly inherited Alzheimer's disease. N Engl J Med. 2012;367(9): 795-804. 
50 Jack CR, Knopman DS, Jagust WJ, Petersen RC, Weiner MW, Aisen PS, et al. Tracking pathophysiological processes in Alzheimer's disease: an updated hypothetical model of dynamic biomarkers. Lancet Neurol. 2013; 12(2):207-16

51 Lambracht-Washington D, Rosenberg RN. Advances in the development of vaccines for Alzheimer's disease. Discov Med. 2013; 15(84):319-26.

52 Schneider JR, Chadee DD, Mori A, Jeanne Romero-Severson DWS. Clinical and psychological characteristics of the initial cohort of the dominantly inherited Alzheimer network (DIAN). Bone. 2008;23(1):1-7.

53 Beach TG, Monsell SE, Phillips LE, Kukull W. Accuracy of the clinical diagnosis of Alzheimer disease at National Institute on aging Alzheimer disease centers, 2005-2010. J Neuropathol Exp Neurol. 2012;71(4):266-73.

54 Knopman DS, Petersen RC, Jack CR. A brief history of "Alzheimer disease": multiple meanings separated by a common name. Neurology. 2019;92(22):1053-9.
55 Reynolds DS. A short perspective on the long road to effective treatments for Alzheimer's disease. Br J Pharmacol. 2019;176(18):363648.

56 Jack CR, Holtzman DM. Biomarker modeling of Alzheimer's disease. Neuron. 2013;80(6): 1347-58.

57 Mo JJ, Li JY, Yang Z, Liu Z, Feng JS. Efficacy and safety of anti-amyloid- $\beta$ immunotherapy for Alzheimer's disease: a systematic review and network meta-analysis. Ann Clin Transl Neurol. 2017;4(12):931-42.

58 Abushakra S, Porsteinsson A, Scheltens S, Sadowsky C, Vellas B, Cummings J, et al. Clinical effects of tramiprosate in APOE4/4 homozygous patients with mild Alzheimer's disease suggest disease modification potential. J Prev Alzheimers Dis. 2017;4(3):149-56.

59 Abushakra S, Porsteinsson A, Vellas B, Cummings J, Gauthier S, Heyet JA, et al. Clinical benefits of tramiprosate in Alzheimer's disease are associated with higher number of APOE4 alleles: the "APOE4 gene-dose effect. J Prev Alzheimers Dis. 2016;3(4):219-28.
60 Tolar M, Abushakra S, Hey JA, Porsteinsson A, Sabbagh M. Aducanumab, gantenerumab, BAN2401, and ALZ-801-the first wave of amyloid-targeting drugs for Alzheimer's disease with potential for near term approval. Alz Res Therapy. 2020;12(1):95.

61 Tolar M, Abushakra S, Sabbagh M. The path forward in Alzheimer's disease therapeutics: reevaluating the amyloid cascade hypothesis. Alzheimers Dement. 2019:1-8.

62 Panza F, Solfrizzi V, Seripa D, Imbimbo BP, Lozupone M, Santamato A, et al. Tau-Centric targets and drugs in clinical development for the treatment of Alzheimer's disease. Biomed Res Int. 2016;2016:3245935.

63 Anti-tau CTOF. Combination therapy of anti-tau and anti-amyloid drugs for disease modification in early-stage Alzheimer's disease: socio-economic considerations modeled on treatments for tuberculosis, HIV/ AIDS and breast cancer. 2020;1-11.

64 Hamelin L, Lagarde J, Dorothée G, Leroy C, Labit M, Comley RA, et al. Early and protective microglial activation in Alzheimer's disease: a prospective study using 18F-DPA-714 PET imaging. Brain. 2016;139(4):1252-64. 